\title{
Sleep duration and cancer risk: time to use a "sleep-years" index?
}

\author{
Thomas C. Erren
}

Received: 21 March 2012/ Accepted: 4 July 2012/Published online: 18 July 2012

(C) Springer Science+Business Media B.V. 2012

\begin{abstract}
With a focus on melatonin, a recent paper in the Journal investigated the hypothesis that endometrial cancer might be associated with the duration, and ultimately, amount of sleep. The authors found that "[s]elfreported sleep duration may not adequately represent melatonin levels." The authors also concluded that there was "weak evidence of an association between sleep duration and endometrial cancer risk." Overall, these are interesting observations because primarily experimental and mechanistic research from many angles supports the study's notion that inappropriate sleep may be a determinant of cancer risk. To find out whether this is so in man, rather than assigning study individuals to fixed or average "baseline sleep categories" i.e., $\leq 5,6,7,8, \geq 9 \mathrm{~h}$ of habitual sleep in the present study, the accumulated amount of sleep over decades should be reconstructed in retrospective or constructed in prospective studies. To achieve this end, future epidemiological studies may want to use a sleep-years index [SYI]. This simple exposure parameter promises to be a sensible, feasible, and affordable way to approximate cumulative time spent at sleep in critical time windows over many years which we should expect to be relevant for the development of cancer. The SYI could be tested and used in observational studies which promise to be comparable and can be merged. This commentary provides roots of the index and explains why and how it
\end{abstract}

T. C. Erren

Institute and Policlinic for Occupational Medicine, Environmental Medicine and Prevention Research,

University of Cologne, Cologne, Germany

T. C. Erren $(\bowtie)$

University Hospital of Cologne, University of Cologne,

Cologne, Germany

e-mail: tim.erren@uni-koeln.de should be used and how it could be interpreted in rigorous studies of biologically plausible links between sleep, on the one hand, and the development of internal cancers, on the other. This commentary also points out limitations of interpreting the SYI. It is emphasized that, where possible, the SYI should be assessed independently of (a) other sleep facets - such as quality - and of (b) known or suspected cancer risk factors. The respective contribution of (a) and (b) to risk must then be assessed during the analyses. Overall, the suggested inclusion and standardization of assessing sleep duration could be an important step forward when evaluating possible cancer risks in relation to sleep. Finally, the proposed approach may prove useful beyond sleep epidemiology per se: to exemplify, research into suggested causal links between disrupted natural sleepwakefulness cycles and increased cancer risks in shiftworkers could also benefit.

Keywords Sleep $\cdot$ Sleep-years $\cdot$ Light $\cdot$ Melatonin · Cancer

"Make everything as simple as possible but not simpler"

Attributed to Einstein.

\section{Introduction}

In the course of the Cologne Symposium 2002 [1], it was predicted $[2,3]$ that sleep duration can be causally related with the development of internal cancers. Dr. Sturgeon et al. [4] now provide the sixth study on the issues concerned and report mixed results. Overall, with regard to how long one sleeps, first [4-8]—but not all [9]—epidemiological evidence could be viewed in line with increased cancer risks in those who sleep less than others [2]. 
Sturgeon et al. [4] inter alia start out from the 2007 International Agency for Research on Cancer (IARC) classification that shift-work, involving circadian disruption, is a probable human carcinogen [10] At the time, 22 international experts identified the following as key experimental and mechanistic evidence for their assessment, namely "[e]xposure to light at night disturbs the circadian system with alterations of sleep-activity patterns, suppression of melatonin production, and deregulation of circadian genes involved in cancer-related pathways" [10]. Remarkably, all this evidence is critically related to the integrity of the "biological night" [10] and, ultimately, to sleep. With further regard to circadian rhythmicity, there are impressive links between manipulating our master clocks, the suprachiasmatic nuclei directly (via electrolytic destruction) [11] or indirectly (via manipulations of the light:dark environments) [12, 13], and the development of cancer in experimental animals. That sleep will be a variable to study with regard to links along the chain of causation which may lead to cancer is also evinced by recent studies [14, 15] which explored the theme from further study angles.

The key question, however, remains:

How can we appropriately test the underlying hypothesis within epidemiological studies which have the advantage to be done in the real world [16] at the cost of being crude when compared with experiments? Put differently:

How could we get more sleep epidemiology with regard to possible cancer endpoints in feasible, informative, and affordable ways?

One candidate approach is to use what was also proposed in Cologne in 2002, namely to employ a sleep-years index [SYI] [2].

\section{The sleep-years index: a simple exposure parameter for epidemiological studies investigating possible links between sleep duration and cancer}

Indeed, relatively "simple" information could be collected - and used - in epidemiological studies designed to detect possible links between sleep duration and cancer developments. Similar to pack-years or so-called fibreyears in cancer studies or for compensation purposes regarding the effects of smoking or asbestos exposure, respectively, information on average hours of sleep in relevant time windows could be collected and parameterized via a sleep-years index: [2] 6, 9, and $12 \mathrm{~h}$ of sleep per day reported for a given calendar year correspond to $1,1.5$, and 2 SYs (when scaling to $6 \mathrm{~h}$ per day of sleep), and these yearly numbers can be accumulated over life time up to the event of interest.

Clearly, wherever possible, studies that employ the SYI must do two additional things: (a) given the very complexity of sleep per se, and our current lack of knowledge, further factors associated with sleep such as its quality, disturbance et cetera should be considered; (b) given the very complexity of the development of cancer, the SYI should be assessed independently of other cancer risk factors, and the respective contribution of all possible determinants of risk must be assessed at the analysis stage of the studies.

\section{Roots and construction of the sleep-years index in the $1940 \mathrm{~s}$}

The underlying idea goes back to research in the late $1940 \mathrm{~s}$. At that time, epidemiologists in the US [17] and the UK [18] independently investigated possible causal links between smoking and lung cancer which were then disputed to say the least. Forty-six years later, Wynder explained that two "favourable facts facilitated" the work [19]. One, there was a strong association between smoking and lung cancer. Two, the information about relevant exposure was easily obtained. In retrospect, exposure assessment was that simple: the researchers asked smokers when they smoked, how much and for how long, and obtained relevant exposure gradients in a straightforward way. Indeed, exposure information which was critical to unmask smoking as the cancer culprit was simply based on the number of cigarettes smoked per day and the number of years which study individuals had smoked ("pack-years" concept thereafter).

This concept of cumulative exposure actually became a cornerstone of modern epidemiology [20, 21]. In practice, the success of such index depends on the ability to estimate exposure intensity (in this case, sleep duration per day) over time. Panel studies asking cohort-study participants repeatedly may be a direct approach to estimate such intensities. These and other basic data may then be used to estimate relative changes of intensity across important covariates like gender, age, and calendar time. Procedures have been developed in occupational epidemiology [20] and other fields [22] which may be adapted appropriately to make optimal use of these data. Backward extrapolations of recent measurements may be performed taking covariates into account [23].

\section{Why develop and use the sleep-years index in future sleep epidemiology?}

Future sleep epidemiology may investigate a biologically plausible role of sleep duration in the development of internal cancers by exploiting four considerations: 
1. The proposed sleep-years index should allow researchers to assess sleep duration in valid, feasible, and affordable ways in populations, be they small or large. In fact, a recent editorial [24] on sleep epidemiology left no doubt that the question "How many hours do you sleep on average?" has been asked in studies many times in the past and offers much prospect for future research and insights. "At present, there are probably more data available for the simple question 'How many hours do you sleep on an average night?' than for any other measure of sleep and much useful work can be achieved using large studies with repeat data for this measure ... it is important to recognize that self-reported sleep duration and disorders are strongly associated with objectively ascertained health outcomes" [24].

2. Rather than assigning study individuals to fixed or average "baseline sleep categories," [4] i.e., $\leq 5,6,7,8$, $\geq 9$ h of sleep in the present study, [4] the accumulated amount of sleep over decades can be reconstructed or constructed in retrospective or prospective studies, respectively. To ask study individuals at the baseline of the Women's Health Initiative (WHI) Observational Study, "About how many hours of sleep did you get in a typical night over the past 4 weeks?" seems to fall short of reliably assessing the sleep length characteristics over many years and decades which should be of interest provided that the hypothesized causal link between sleep duration and the development of cancers were there. Empirically, Sturgeon et al. noted that "a total of $59 \%$ of the study population reported the same sleep patterns at year 3 of follow-up as baseline." Importantly, the habitual duration of sleep in individuals-certainly more often than not-can and will change over time. With the use of SYs, we will likely obtain significant gradients of cumulative time spent at sleep, offering a promising data base for epidemiological studies into the hypothesized links between varying sleep duration and cancer.

3. The rationale for the SYI and its straightforwardness may appeal to many researchers. It could thus be a recipe to avoid numerous future studies which employ studyspecific, and ultimately incomparable, exposure assessments of sleep duration.

4. The suggested SYI would make sense also with regard to the sustainability of the approach. Dyson's recipe for success of sustainable scientific advances comes to mind here: research projects may be(come) sustainable, if their very costs make them candidates for follow-up studies rather than limiting them to one- or two-time enterprises. In this very vein, what Dyson [25] wrote with regard to projects being sustainable or not may apply to methodological approaches as well: "A project is sustainable if it is cheap enough to be the first of a series continuing indefinitely into the future."

\section{How to use and interpret the SYI? [2]}

- How would we obtain the relevant information on sleep?

We could do so via interviews of study individuals, seeking information on average hours of sleep during relevant periods of time preceding the development of cancers over years and sometimes decades. And we may develop exposure models that measure the variation of such intensity estimates across relevant covariates and use such models to estimate the sleepyears index from incomplete information.

- How would we practically implement the SYI concept in epidemiological studies?

Being a way to differentially measure the amount a person has slept over a long period of time, the SYI is calculated by multiplying the number of hours of sleep per day by the number of years the person reconstructs sleep duration. For example, 1 sleepyear is scaled to be equal to sleeping $6 \mathrm{~h}$ per day for 1 year [2], or $12 \mathrm{~h}$ per day for half a year, and so on. To illustrate the principle:

\begin{tabular}{|c|c|c|c|c|c|c|}
\hline \multicolumn{5}{|c|}{ Hours of sleep/day/year } & \multicolumn{2}{|c|}{ Sleep-year (SY) } \\
\hline \multicolumn{5}{|l|}{5} & \multicolumn{2}{|l|}{0.83} \\
\hline \multicolumn{5}{|l|}{6} & \multicolumn{2}{|l|}{1} \\
\hline \multicolumn{5}{|l|}{7} & \multicolumn{2}{|l|}{1.17} \\
\hline \multicolumn{5}{|l|}{8} & \multicolumn{2}{|l|}{1.33} \\
\hline \multicolumn{5}{|l|}{9} & \multicolumn{2}{|l|}{1.5} \\
\hline \multicolumn{5}{|l|}{10} & \multicolumn{2}{|l|}{1.67} \\
\hline \multicolumn{5}{|l|}{11} & \multicolumn{2}{|l|}{1.83} \\
\hline \multicolumn{5}{|l|}{12} & \multicolumn{2}{|l|}{2} \\
\hline \multirow[b]{2}{*}{ Age } & \multicolumn{4}{|c|}{ Hours of sleep/day/year } & \multirow{2}{*}{\multicolumn{2}{|c|}{$\begin{array}{l}\text { Accumulated SYs during } \\
4 \text { decades }\end{array}$}} \\
\hline & $20-29$ & $30-39$ & $40-49$ & $50-59$ & & \\
\hline I1 & 6 & 8 & 8 & 7 & $10+13+13+12$ & 48 \\
\hline $\mathrm{I} 2$ & 8 & 8 & 8 & 8 & $13+13+13+13$ & 52 \\
\hline I3 & 5 & 6 & 6 & 5 & $8+10+10+8$ & 36 \\
\hline I4 & 9 & 8 & 8 & 6 & $15+13+13+10$ & 51 \\
\hline
\end{tabular}

In this example, Individuals 1-4 have accumulated the \# of SYs as indicated in bold print in the right column. Assuming that you have incident breast 
cancer cases which are diagnosed at the age of (1) 40 , (2) 50, and (3) 60 years of age:

Under scenario (1): I1-4 would have accumulated 23, 26, 18, and $28 \mathrm{SYs}$, respectively.

Under scenario (2): I1-4 would have accumulated 36 , 29, 28, and 41 SYs, respectively.

Under scenario (3): I1-4 would have accumulated 48, 52, 36, and $51 \mathrm{SYs}$, respectively.

- What dose-response relationships are we likely to observe?

While the SYI starts out from cumulative exposure approaches such as for smoking (pack-years) and asbestos (fibre-years), it is - on theoretical and albeit limited empirical grounds-biologically plausible that relationships between sleep-years and cancer evince higher risks on both exposure ends and form bell-shaped curves. Namely, populations with shorter or longer sleep durations may have higher cancer risks. In this vein, it is important to note that the relationship between SY and cancer risk is unlikely to follow some linear dose-response relationship as smoking and asbestos exposures do. In fact, the limited empirical evidence we have to date shows an increased mortality in both short and long sleepers [26-30].

- How would we interpret the lack of an association between SYs and the development of cancer?

Admittedly, not detecting associations would not necessarily exonerate the notion that links between facets of sleep and carcinogenesis are at work. After all, the SYI may not be sensitive enough to the critical aspects of sleep, such as its very quality in the time window of interest preceding the development of cancer.

- How would we interpret an association between SYs and the development of cancer?

Demonstration of significant correlations between SYs and cancer could imply that we may be looking at real effects which may have a significant impact on public health.

\section{Why use the sleep-years index when researching possible links between sleep and cancer: and beyond?}

With final regard to Dr. Sturgeon et al. [4], she and her team suggest that future studies should utilize "urinary levels of melatonin in relation to endometrial cancer risk" [4]. This approach is fine in principle. And yet, melatonin on its own, and in urine alone, may not be easily and readily interpretable either [31] To exemplify, note-as possible complications- that there are individuals who have a genetically weak or strong machinery and produce low or high melatonin levels at night; [31] moreover, there are circadian changes in melatonin receptor density or sensitivity [32], and some of the actions postulated for melatonin involve receptors while other actions do not [31].

Therefore, it should not surprise that Sturgeon et al. concluded that "[s]elf-reported sleep duration may not adequately represent melatonin levels." But, importantly, the authors also concluded that "[they] found weak evidence of an association between sleep duration and endometrial cancer risk."

To try to find out whether this is really the case, we may want to use the proposed sleep-years index as a sensible, feasible, and affordable way to approximate cumulative time spent at sleep. Remarkably, the SYI approach could be used in both retrospective and prospective observational studies which may be comparable and ultimately merged. Moreover, this approach could be useful beyond sleep studies per se. To provide but one example, studies for which Drs. Haus and Smolensky called in a widely cited article [33] in the Journal to investigate suggested causal links between disrupted natural sleep-wakefulness cycles and an increased cancer incidence in shift-workers could also benefit from the SYI approach.

Overall, introducing the SYI to epidemiological research has to be balanced between two scales: the sleepyears index could be, on the one hand, tantalizing because it promises a simple and standardized approach to evaluate possible cancer risks in relation to sleep duration. On the other hand, there is the peril to over-simplify both, the complex exposure "sleep" and the complex disease endpoint "cancer", which may lead to the misinterpretation of possible cause-effect relationships. To avoid the latter, wherever possible, further facets of sleep—such as sleep quality - and of established or suspected risk factors for cancer must be collected and considered as diligently as possible as a prerequisite for the SYI to become an informative approach for epidemiological cancer research in particular and chronic disease research in general.

Acknowledgments The author acknowledges insightful suggestions by anonymous reviewers and by Peter Morfeld considered for the printed manuscript.

Conflict of interest The author declares that he has no conflict of interest.

\section{References}

1. Erren TC, Piekarski C (2002) Light and life-facts and research perspectives at the Cologne Light Symposium 2002. Neuro Endocrinol Lett 23(Suppl 2):4-6 
2. Erren TC (2002) Does light cause internal cancers? The problem and challenge of an ubiquitous exposure. Neuro Endocrinol Lett 23(Suppl 2):61-70

3. Stevens RG (2002) Lighting during the day and night: possible impact on risk of breast cancer. Neuro Endocrinol Lett 23(Suppl 2):57-60

4. Sturgeon SR, Luisi N, Balasubramanian R, Reeves KW (2012) Sleep duration and endometrial cancer risk. Cancer Causes Control. Feb 242012

5. Verkasalo PK, Lillberg K, Stevens RG et al (2005) Sleep duration and breast cancer: a prospective cohort study. Cancer Res 65:9595-9600

6. Kakizaki M, Kuriyama S, Sone T et al (2008) Sleep duration and the risk of breast cancer: the Ohsaki Cohort Study. Br J Cancer 99:1502-1505

7. Kakizaki M, Inoue K, Kuriyama S et al (2008) Sleep duration and the risk of prostate cancer: the Ohsaki Cohort Study. Br J Cancer 99:176-178

8. Wu AH, Wang R, Koh WP, Stanczyk FZ, Lee HP, Yu MC (2008) Sleep duration, melatonin and breast cancer among Chinese women in Singapore. Carcinogenesis 29:1244-1248

9. Pinheiro SP, Schernhammer ES, Tworoger SS, Michels KB (2006) A prospective study on habitual duration of sleep and incidence of breast cancer in a large cohort of women. Cancer Res 66:5521-5525

10. Straif K, Baan R, Grosse Y et al (2007) Carcinogenicity of shiftwork, painting, and firefighting. Lancet Oncol 8:1065-1066

11. Filipski E, King VM, Li X et al (2002) Host circadian clock as a control point in tumor progression. J Natl Cancer Inst 94:690-697

12. Filipski E, Delaunay F, King VM, Wu MW, Claustrat B, Grechez-Cassiau A, Guettier C, Hastings MH, Francis L (2004) Effects of chronic jet lag on tumor progression in mice. Cancer Res 64:7879-7885

13. Filipski E, Innominato PF, Wu M, Li XM, Iacobelli S, Xian LJ, Levi $F$ (2005) Effects of light and food schedules on liver and tumor molecular clocks in mice. J Natl Cancer Inst 97:507-517

14. Kang TH, Lindsey-Boltz LA, Reardon JT, Sancar A (2010) Circadian control of XPA and excision repair of cisplatin-DNA damage by cryptochrome and HERC2 ubiquitin ligase. Proc Natl Acad Sci USA 107:4890-4895

15. Kripke DF, Langer RD, Kline LE (2012) Hypnotics' association with mortality or cancer: a matched cohort study. BMJ Open 2:e00850

16. Doll R (1994) Introduction and overview. In: Samet JM (ed) Epidemiology of lung cancer. Lung biology and health and disease, vol 74. Marcel Dekker Inc., New York
17. Wynder EL, Graham EA (1950) Tobacco smoking as a possible etiologic factor in bronchiogenic carcinoma; a study of 684 proved cases. J Am Med Assoc 143:329-336

18. Doll R, Hill AB (1950) Smoking and carcinoma of the lung; preliminary report. Br Med J 2:739-748

19. Wynder EL (1996) Invited commentary: response to Science article, "Epidemiology faces its limits". Am J Epidemiol 143:747-749

20. Checkoway H, Pearce N, Kriebel D (2004) Research methods in occupational epidemiology, vol 34, 2nd edn. Oxford University Press, New York

21. Rothman KJ, Greenland S, Lash TL (2008) Modern epidemiology, 3rd edn. Lippincott Williams \& Wilkins, Philadelphia

22. White E, Armstrong BK, Saracci R (2008) Principles of exposure measurement in epidemiology, 2nd edn. Oxford University Press, Oxford

23. Ignacio JS, Bullock WH (2006) A strategy for assessing and managing occupational exposures, 3rd edn. AIHA, Fairfax

24. Ferrie JEKM, Salo P, Singh-Manoux A, Kivima M (2011) Sleep epidemiology $-\mathrm{a}$ rapidly growing field. Int $\mathrm{J}$ Epidemiol 40:1431-1437

25. Dyson F (1999) The sun, the genome, and the internet: tools of scientific revolution (New York Public Library Lectures in Humanities), Oxford University Press

26. Patel SR, Ayas NT, Malhotra MR et al (2004) A prospective study of sleep duration and mortality risk in women. Sleep 27:440-444

27. Youngstedt SD, Kripke DF (2004) Long sleep and mortality: rationale for sleep restriction. Sleep Med Rev 8:159-174

28. Amagai Y, Ishikawa S, Gotoh T et al (2004) Sleep duration and mortality in Japan: the Jichi Medical School Cohort Study. J Epidemiol 14(4):124-128

29. Lan TY, Lan TH, Wen CP et al (2007) Nighttime sleep, chinese afternoon nap, and mortality in the elderly. Sleep 30(9):11051110

30. Gallicchio L, Kalesan B (2009) Sleep duration and mortality: a systematic review and meta-analysis. J Sleep Res 18(2):148-158

31. Erren TC, Reiter RJ, Piekarski C (2003) Chronodisruption and melatonin: the need for sensible exposure metrics in epidemiological studies. J Pineal Res 45:335-336

32. Dubocovich ML, Markowska M (2005) Functional MT1 and MT2 melatonin receptors in mammals. Endocrine 27:101-110

33. Haus E, Smolensky M (2006) Biological clocks and shift work: circadian dysregulation and potential long-term effects. Cancer Causes Control 17:489-500 